Cite this: Soft Matter, 2014, 10, 1151

\title{
Optimal ionic strength for nonionically initiated polymerization $\uparrow$
}

\begin{abstract}
Marta E. Dobrowolska and Ger J. M. Koper*
Surfactant-free emulsion polymerization involving a nonionic, and hence uncharged initiator presents a new approach towards environmentally friendly procedures to synthesize latex particles. Under optimal solvent conditions, notably $\mathrm{pH}$ and ionic strength, the latex particles are stabilized by the natural development of ionic charge at the surface of the particles. We emphasize that the present process does not at all involve the addition of stabilizers such as surfactants or the creation of surface-active species from ionic initiators. The width of the size distribution is found to vary strongly with experimental conditions, notably the ionic strength and to a much lesser extent $\mathrm{pH}$. The phenomenon is explained by a critical ionic strength dependence of the aggregation of the just nucleated primary particles into larger secondary particles, the so-called "coagulative nucleation" step.
\end{abstract}

Received 23rd July 2013

Accepted 27th November 2013

DOI: $10.1039 / \mathrm{c} 3 s m 51998 \mathrm{~h}$

www.rsc.org/softmatter

typically lead to a high size polydispersity of the resulting latex.

\section{Introduction}

Emulsion polymerization is a widely used process for the production of an extensive range of polymers for many applications. ${ }^{1-3}$ Control over size and polydispersity in any of these applications is highly called for and in order to achieve this, typical formulations rely on the stabilization induced by surface active agents. Such surfactants are believed to adsorb on the water-polymer interface of the particles during and after their synthesis in order to provide protection against coagulation and subsequent coalescence. Nonionic surfactants provide steric stabilization and ionic surfactants provide electrostatic stabilization. ${ }^{1}$ At the same time, surfactants are the most significant contamination of the final product and there have been many attempts to reduce their use and provide rules for surfactantfree processes. ${ }^{4}$ In such surfactant-free formulations one only employs monomer, initiator and water where the initiator can be both oil-soluble and water-soluble. Ionic initiators are successfully used in such formulations but in the end do not differ in quality from the conventional surfactant-rich formulations. This is because the hydrophilic, usually ionic, group gets incorporated in the polymer chains to subsequently act as a stabilizing surface agent of the polymer particles. The only important formulation aspect is that the final surface concentration of ionic groups needs to be sufficient to provide the required surface charge for stability. ${ }^{5}$ There are, however, some formulations that involve oil-soluble initiators for which there is no such stabilizing mechanism known. ${ }^{6,7}$ These formulations

Delft University of Technology, Department of Chemical Engineering, Julianalaan 136, 2628BLDelft,The Netherlands.E-mail:m.e.dobrowolska@tudelft.nl; g.j.m.koper@ tudelft.nl; Tel: +31 (0)15 2788218

$\dagger$ Electronic supplementary information (ESI) available. See DOI: $10.1039 / \mathrm{c} 3 \mathrm{sm} 51998 \mathrm{~h}$
The intriguing question that we intend to answer here concerns the stabilization mechanism and the associated formulation window.

What is known - albeit not understood - is that hydrophobic surfaces get charged if exposed to water. ${ }^{8}$ Already in the $19^{\text {th }}$ century it was observed that air bubbles in water are negatively charged so that in an electrophoretic experiment these migrate towards the positive electrode. ${ }^{9}$ More recently, ${ }^{10}$ studies investigating the zeta potential of air bubbles under a wide range of $\mathrm{pH}$ values show that these are negatively charged and display an isoelectric point at a $\mathrm{pH}$ value of about 4 . Below that $\mathrm{pH}$ value the bubbles become positively charged. The interface of neutral water with hydrophobic surfaces, such as hydrocarbon oils, is similar to the interface between air and water, and it has long been known that such interfaces also acquire a negative charge when the water is of neutral $\mathrm{pH} \cdot{ }^{\mathbf{1 1 , 1 2}}$ Similar to the situation of interfaces between air and water, various authors ${ }^{\mathbf{1 3 , 1 4}}$ have proven that the point of zero charge for hydrophobic surfaces in contact with water is between $\mathrm{pH} 3$ and 4 . As pointed out by Beattie et al. ${ }^{13}$ hexadecane-in-water emulsions prepared at $\mathrm{pH} 7$ are stable for several hours. In order to maintain the $\mathrm{pH}$ while refining such an alkane-in-water emulsion, additional hydroxide is required. Based on Grahame's equation and Stern's isotherm, Marinova et al. ${ }^{15}$ modeled the behavior of the zeta potential as measured for oil droplets in water as a function of $\mathrm{pH}$ and ionic strength of the aqueous solution. By fitting the model to experimental data, they obtained reasonable model parameter values such as for the equilibrium constant for hydroxyl adsorption. Recently, Roger et al., ${ }^{\mathbf{1 6}}$ suggested that the negative surface charge is originating from unreacted traces of fatty acids dissolved in the oil. However, the authors did not convince the scientific community and in actual fact presented 
another piece of evidence for the presence of hydroxide ions at the interfaces. ${ }^{17}$

In a preliminary experiment, we verified that styrene-inwater emulsions behave according to the predictions of Marinova et al. ${ }^{15}$ An important result is that the observations are independent of particle size at least up to micrometers. More importantly, the ionic strength dependence of the emulsion stability followed classical DLVO theory predictions. ${ }^{18,19}$ Recently, Yamamoto demonstrated the synthesis of polystyrene, micron-sized particles using a non-ionic initiator in a soap-free environment. ${ }^{6}$ This author attributed the origin of the stabilizing negative charge to the polarization of the electron-active functional groups decomposed from the initiators and the pielectron cloud of the benzene ring in styrene. However, this is not very likely to be the case because the concentrations of the monomer and initiator used should lead to stabilization at much smaller particle sizes as follows from a simple geometrical analysis. ${ }^{20}$

Now, having tentatively established the stabilization mechanism, we next turn to a more detailed study on the conditions under which surfactant-free emulsion polymerization of styrene with a non-ionic initiator can be performed. In order to unequivocally illustrate this effect, we use a very low solid content of styrene.

\section{Materials and methods}

Polystyrene particles were synthesized by surfactant-free emulsion polymerization. We closely followed the procedure by Tauer et al. ${ }^{21}$ so that more information about the development of the system, which is not the subject of this paper, can be found. A given amount of sodium chloride (Sigma Aldrich, $\geq 99.5 \%$ ) was added to $100 \mathrm{~mL}$ of MilliQ water in a three-neck glass flask. The solution was bubbled with $\mathrm{N}_{2}$ and degassed with vacuum; the procedure was repeated 3 times while stirring (150 rpm). Subsequently, $0.68 \mathrm{~g}$ of styrene (Sigma Aldrich, $\geq 99 \%$ ) was injected into the flask. Prior to that step, the protective inhibitor was removed from the styrene using a prepacked column (Sigma Aldrich, for removal tert-butylcatechol). The solution was equilibrated in a closed flask for $2 \mathrm{~h}$ at $70^{\circ} \mathrm{C}$. Polymerization was started by injection of an initiator, $0.019 \mathrm{~g}$ azobisisobutyronitrile (AIBN) dissolved in $0.23 \mathrm{~g}$ styrene. The solution was then allowed to polymerize under nitrogen conditions for $5 \mathrm{~h}$. During the polymerization a portion of the reaction solution was taken out after 90 and 180 minutes under the flow of inert gas $\left(\mathrm{N}_{2}\right)$ with a gas tight syringe and freezedried under high vacuum. A part of the dried material was put on a specimen holder and subsequently a molecular layer of gold was deposited for analysis by Scanning Electron Microscope (FEI/Philips XL30 SFEG) operating at $5 \mathrm{kV}$ and using the Ultra-High Resolution mode (UHR-SEM).

Water-suspended samples were analyzed by dynamic light scattering (DLS, Zetasizer Nano ZS, Malvern). For sample quality, the polydispersity index (PDI) as calculated by the instrument is defined as the coefficient of variation of the $z$-averaged mean particle size and is obtained from a cumulant expansion of the measured electric field autocorrelation function (see ref. 5 Section 5.8). The PDI is dimensionless and scaled such that values smaller than 0.05 are rarely seen other than with highly monodisperse standards. Values greater 0.7 indicate that the sample has a very broad size distribution and is probably not suitable for DLS sizing. The various size distribution algorithms work with the data that falls between these two extremes. The calculations for these parameters are defined in the standard documents 13321:1996 E and 22412:2008 (ISO, Geneva, Switzerland).

\section{Results}

Let us first consider the situation at the moderate ionic strength of $1 \mathrm{mM}$. Before starting the polymerization, the aqueous phase is a dilute emulsion with rather large monomer droplets of about $400 \mathrm{~nm}$ as can be seen from the Fig. S1 $\uparrow$ in the Supporting Information. The size distribution of this emulsion is quite broad and the aqueous phase is saturated with monomer. After addition of the initiator dissolved in an aliquot of monomer, polymerization starts and the observed particle size steeply decreases and subsequently slowly grows with polymerization time. From the micrographs in Fig. 1 we learn that the emulsion polymerization process conducted here clearly involves a coagulation step where primary particles aggregate into larger secondary particles. ${ }^{22,23}$ The coagulation rate, growth rate, and final particle size are determined by solvent conditions as will be discussed below. Under the present conditions of $\mathrm{pH} 8$ and ionic strength $1 \mathrm{mM}$, the zeta potential of the aggregates has been measured to be around $-50 \mathrm{mV}$ and hardly varies with the initiator concentration. However under different conditions, notably at higher ionic strength, strong variations in zeta potential are visible when the particles are fully grown (see ESI). $\dagger$

By varying the $\mathrm{NaCl}$ concentration between $10^{-7} \mathrm{M}$ and $10^{-2}$ $\mathrm{M}$, the effect of the emulsion phase ionic strength on the size distribution was studied in more detail. It was observed that as the ionic strength increased, the size distribution became narrower up until a concentration of $10^{-3} \mathrm{M}$. At higher concentrations the synthesized particles were becoming more polydisperse and in addition were becoming larger. The scanning electron micrographs in Fig. 2 clearly suggest that the quality of the particle dispersion strongly varies with the ionic strength. As was discussed above, it has also been seen that the

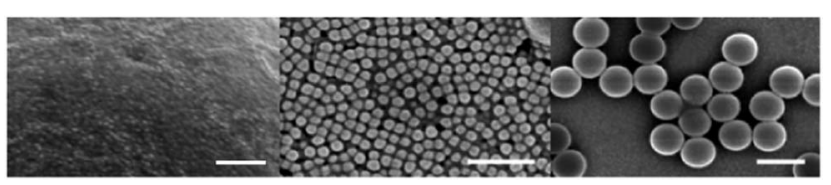

Fig. 1 UHR-SEM micrographs of samples taken at three instants after initiation of the polymerization process done at $1 \mathrm{mM} \mathrm{NaCl}$ concentration. The left micrograph was taken after 15 min of polymerization time shows primary particles. The middle micrograph was taken after 90 min of polymerization time clearly shows that particles are small aggregates of primary particles. The right micrograph was taken after 180 min polymerization time and shows the relatively homogeneous final particles. The scale bars are $200 \mathrm{~nm}$. 

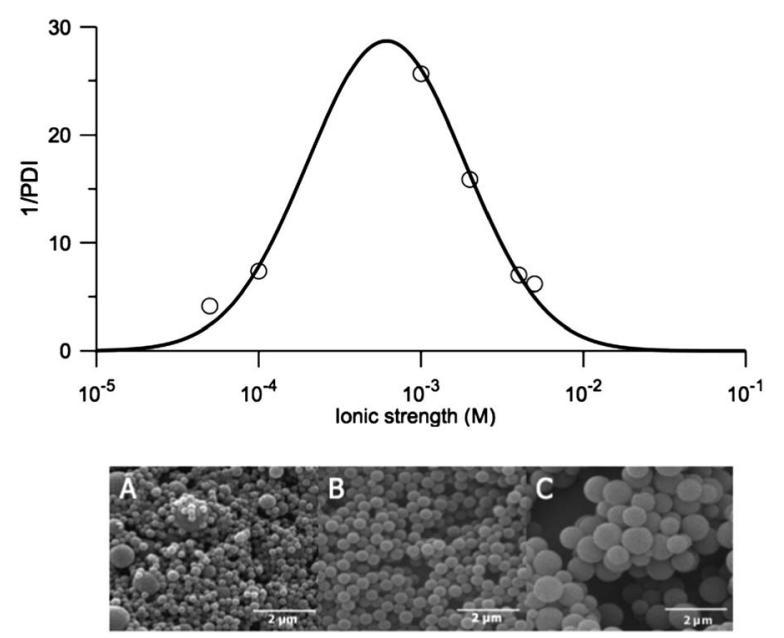

Fig. 2 Optimal conditions as determined by the polydispersity of polystyrene latex particles as a function of ionic strength; the line serves as a guide to the eye. Scanning electron micrographs of polystyrene particles polymerized with no additional salt (A), with $1 \mathrm{mM}$ $\mathrm{NaCl}(\mathrm{B})$ and $5 \mathrm{mM} \mathrm{NaCl}(\mathrm{C})$.

zeta potential, which is a measure of the colloidal stability of the samples, also varies with ionic strength and only below $1 \mathrm{mM}$ a moderate surface charge has been measured. Other types of monovalent salts, such as $\mathrm{KCl}$, resulted in very a similar behavior compared to what is reported here. To quantify the results, we have defined a quality factor as the inverse of the polydispersity index (PDI) calculated from the size distribution of the samples as obtained from the emulsion polymerization experiments at various values of ionic strengths (see Materials and methods).

We stress that the quality factor as defined here is based on the idea that a shallow size distribution is optimal. There are of course situations where a broad size distribution is optimal in which case a better definition of a quality factor would be the PDI itself. The results of our analysis have been plotted as a function of ionic strength in Fig. 2. An optimum around $1 \mathrm{mM}$ is clearly visible from the graph albeit that the line just serves as a guide to the eye. Therefore in the following discussion we will assume the ionic strength of $1 \mathrm{mM}$ to be the optimal one that we have determined.

\section{Discussion}

As discussed in the Introduction, the commonly held view is that hydrophobic surfaces become negatively charged in aqueous environments, which is assumed to be due to the adsorption of hydroxide ions that are naturally present in water. To ensure reproducible conditions, the solvent is adjusted to be slightly basic with a pH of at least 8 . Hydrophobic particles thus acquire a zeta potential that does not increase above $-30 \mathrm{mV}$ at ionic strengths lower than $10 \mathrm{mM} \cdot{ }^{15}$ Even if the mechanism in itself is not known in detail, the experimental facts clearly indicate that the charge development both can be deduced from electrophoretic mobility measurements ${ }^{15}$ as well as from stability experiments. ${ }^{13}$
Let us therefore review the effect of ionic strength on the quality of the latex dispersions in some more detail. As is known from electrolyte theory, see for example the treatment in the monograph by J. Israelachvili, ${ }^{24}$ the ionic strength mainly affects the thickness of the double layer, which is the region within which the surface potential decays to vanish beyond. For larger separations than the double layer thickness, particles do not interact with one another and hence only when the double layers of two particles are overlapping there is a significant, initially repulsive interaction. Upon closer approach, the particles are attracted by van der Waals forces and subsequently attach irreversibly to each other. The latter process is called flocculation and when the particles are soft they subsequently merge or coalesce. The thickness of this double layer varies inversely with the square root of the ionic strength and for a monovalent electrolyte its characteristic Debye length scale amounts to $3 \mathrm{~nm}$ at $10 \mathrm{mM}$ and becomes about $30 \mathrm{~nm}$ for $0.1 \mathrm{mM}$. The typical distance between particles depends on their concentration as well as on their size. As a consequence, these are then also parameters in the flocculation rate of the particles ${ }^{5}$ in addition to the barrier height in the repulsive interaction.

In a surfactant free emulsion polymerization process there are two steps where aggregation can take place, see Fig. 1 of ref. 23. The first is where the primary particles, which nucleated from the insoluble oligomeric chains, coagulate into secondary particles, the so-called "coagulative nucleation" step. ${ }^{23,25}$ The second step is the, usually undesired, aggregation of secondary particles into even larger structures. After nucleation, the primary particles continue to grow by polymerization and under favorable conditions aggregate to form secondary particles. The final size distribution of the secondary particles is, again under favorable conditions, relatively sharp. In the optimal case of $1 \mathrm{mM}$ ionic strength, the primary particles are relatively highly charged which would normally guarantee good colloidal stability. Despite being highly charged, these particles do aggregate. It has recently been argued by Herrera-Ordonez et $a .^{26}$ that attachment occurs through termination of active chains on either surface of colliding particles. Similar arguments have already been put forward by Tauer et al. in the early nineties. ${ }^{27}$ Such attachment is partially reversible due to the fact that the chains are still relatively short and can hence easily desorb from one of the particles. This makes the size of the secondary particles kinetically controlled by a balance between the electrostatically controlled clustering of primary particles and the rate of particle detachment. The latter process is determined by the mobility of chains in the clustered particles and not by solution parameters like ionic strength so that the latter indeed becomes the control parameter for the particle size distribution. As argued previously, ${ }^{22}$ a partially reversible process accounts for the experimentally observed stationary particle size distribution with increasing median value as for instance discussed by Privman. ${ }^{28}$

The observed effect of ionic strength can now be rationalized as follows. Too high an ionic strength results in a thin double layer, which always leads to flocculation. In addition, it reduces the repulsive barrier. As a consequence, the primary particles have no chance to form reversible bonds; they immediately are 
irreversibly flocculated by means of the van der Waals forces. Also clusters of primary particles can participate in this coagulation process, which leads to a broad particle size distribution. At optimal ionic strength the particles can approach close enough to have a reasonable chance to bind through termination of active chains on either particle. However, at very low ionic strength this process is inhibited and the reversible aggregation into secondary particles does not take place. Instead, the primary particles will continue to grow. As recently discussed by Privman, ${ }^{28}$ this prolonged growth always leads to broad size distributions. In addition, due to fluctuations in surface charge and active chain length, some particles still aggregate albeit most often irreversibly which leads to coalescence and hence even broader size distributions.

Similar experiments as discussed here were performed by Yamamoto $^{6}$ who focussed more on size than on dispersion quality. The ionic strength variation in this case was brought about changing the valence of the counter-ion in chloride compounds, which leads to the exploration of a much smaller range of ionic strength values than done here. Hence, our result for AIBN will also hold for all other nonionic initiators as long as they do not have a strong influence on the surface properties of the latex particles in aqueous suspensions.

\section{Conclusions}

In conclusion, we have shown that surfactant-free emulsion polymerization can be achieved with non-ionic initiators while maintaining good dispersion quality of the final result. This allows one to circumvent the use of ionic initiators that lead to hydrophilic surface-active species that deteriorate the final product. In order to obtain the optimal result, the solvent quality has to be adapted. In the present case this largely affects the ionic strength and hardly the $\mathrm{pH}$. This is the case, because the phenomenon uses the apparent accumulation of hydroxyl ions at the solid- water interface, which is relatively insensitive to acidity. Interestingly, the required ionic strength is very comparable to what is employed for the same emulsion polymerization experiment performed with potassium persulfate (KPS). ${ }^{23}$

\section{Acknowledgements}

We acknowledge financial support from the Dutch Polymer Institute (DPI) and COST CM1101 Action.

\section{Notes and references}

1 R. H. Ottewill and J. M. e. Asua in, Polymeric Dispersions: Principles and Applications, Kluwer Academic Publishers, Dordrecht, 1997.

2 P. A. Lovell and M. S. El-Aasser, Emulsion Polymerization and Emulsion Polymers, Wiley, Chichester, England, 1997.
3 D. Urban and K. Takamura, Polymer Dispersions and Their Industrial Applications, Wiley-VCH, Wienheim, Germany, 2002.

4 K. Tauer, R. Deckwer, I. Kühn and C. Schellenberg, Colloid Polym. Sci., 1999, 277, 607-626.

5 R. R. Paul and C. Hiemenz, Principles of Colloid and Surface Chemistry, Third Edition, Revised and Expanded, CRC Press, 1997.

6 T. Yamamoto, Colloid Polym. Sci., 2012, 290, 1023-1031.

7 M. J. Francis, T. K. Boyson and R. M. Pashley, Colloids Surf., A, 2008, 328, 44-51.

8 L. S. McCarty and G. M. Whitesides, Angew. Chem., Int. Ed., 2008, 47, 2188-2207.

9 G. Quincke, Annu. Phys. Rev., 1861, 113, 513-598.

10 M. Takahashi, J. Phys. Chem. B, 2005, 109, 21858-21864.

11 J. C. Carruthers, Trans. Faraday Soc., 1938, 34, 0300-0307.

12 W. Dickinson, Trans. Faraday Soc., 1941, 37, 0140-0147.

13 J. K. Beattie and A. M. Djerdjev, Angew. Chem., Int. Ed., 2004, 43, 3568-3571.

14 T. W. Healy and D. W. Fuerstenau, J. Colloid Interface Sci., 2007, 309, 183-188.

15 K. G. Marinova, R. G. Alargova, N. D. Denkov, O. D. Velev, D. N. Petsev, I. B. Ivanov and R. P. Borwankar, Langmuir, 1996, 12, 2045-2051.

16 K. Roger and B. Cabane, Angew. Chem., Int. Ed., 2012, 51, 5625-5628.

17 J. K. Beattie and A. Gray-Weale, Angew. Chem., Int. Ed., 2012, 51, 12941-12942.

18 E. J. W. Verwey, J. Th. G. Overbeek, Theory of the stability of lyophobic colloids, Elsevier, Amsterdam, 1948.

19 B. V. Derjaguin and L. Landau, Acta Physicochim. URSS, 1941, 14, 633-662.

20 D. F. Evans and H. Wennerstrom, The Colloidal Domain, VCH, New York, 1994.

21 P. Nazaran and K. Tauer, Macromol. Symp., 2007, 259, 264273.

22 P. J. Feeney, D. H. Napper and R. G. Gilbert, Macromolecules, 1984, 17, 2520-2529.

23 M. E. Dobrowolska, J. H. van Esch and G. J. M. Koper, Langmuir, 2013, 29, 11724-11729.

24 J. Israelachvili, Intermolecular \& Surface Forces, Elsevier, 2007.

25 P. J. Feeney, D. H. Napper and R. G. Gilbert, Macromolecules, 1987, 20, 2922-2930.

26 J. Herrera-Ordonez, O. Rivera, H. Maldonado-Textle and J. C. Ramirez, J. Polym. Sci., Part A: Polym. Chem., 2005, 43, 1963-1972.

27 K. Tauer, G. Reinisch, H. Gajewski and I. Muller, J. Macromol. Sci., Part A: Pure Appl. Chem., 1991, 28, 431-460.

28 V. Privman, in Interdisciplinary Transport Phenomena: Fluid, Thermal, Biological, Materials, and Space Sciences, ed. S. S. Sadhal, Blackwell Publishing, Oxford, 2009, vol. 1161, pp. 508-525. 